5 a Department of Environmental Engineering, Peking University, Key Laboratory

\section{Spectroscopic Characterization of Changes of DOM Deprotonation-protonation Properties in Water Treatment Processes}

Mingquan Yan a, *, Deborah Dryer b and Gregory V. Korshin c of Water and Sediment Sciences, Ministry of Education, Beijing 100871, China b Hazen \& Sawyer, 498 Seventh Avenue, New York, NY 10018, United States;

c Department of Civil and Environmental Engineering, University of Washington, Seattle, WA 98195-2700 United States

* Corresponding author: Mingquan Yan, Department of Environmental Engineering, College of Environmental Sciences and Engineering, Peking University, Beijing 100871, China; Tel: +86 10 62755914-81, Fax: +86 10 62756526. E-mail: yanmq@pku.edu.cn 


\section{Abstract}

The deprotonation-protonation properties of dissolved organic matter (DOM) in drinking water produced at critical treatment points were quantified using absorbance spectra in combination with DOM fractionation data. Analysis of differential spectra of DOM present in inlet, settled and filtered waters from two large treatment plants and their fractions were obtained. The data demonstrated the presence of six Gaussian bands largely associated with carboxylic and phenolic DOM functionalities. Properties of the protonation-active groups of DOM in raw and treated waters were further examined based on data of potentiometric titrations at $\mathrm{pH}$ from 2.5 to 10 . Interpretation of the differential log-transformed absorbance at wavelength 350 $\mathrm{nm}\left(\mathrm{DInA}_{350}\right)$ based on the NICA-Donnan model showed that the normalized concentrations of low- and high-affinity protonation-active groups in residual DOMs increases as a result of water treatment. This was consistent with the results of DOM fractionation. This study demonstrates that changes of the composition and reactivity of DOM found in drinking water treatment sequences can be quantified based on the examination of their optical properties.

Keywords: absorbance, dissolved organic matter (DOM), NICA-Donnan model, protonation, fractionation, drinking water treatment 


\section{Introduction}

Dissolved organic matter (DOM) is ubiquitous in aquatic systems. It affects color, taste and odor and generation of disinfection by-products (DBPs) in drinking water (Leenheer and Croué, 2003; Richardson et al., 2007; Ding and Zhang, 2009; Richardson and Ternes, 2011). DOM also facilitates the transport of many heavy metals and hydrophobic pollutants and increases coagulant demand (Croue et al., 2000; Liu et al., 2007; Yan et al., 2008; Park, 2009; Yan et al., 2013a).

DOM fractionation by XAD8, XAD4 and other resins (Thurman and Malcolm, 1981; Croue et al., 2000; Muller et al., 2000; Schafer et al., 2002; Croue et al., 2003) and structure-sensitive methods such as nuclear magnetic resonance (NMR), pyrolysis GC/MS, Carbon K-edge Near Edge X-Ray Absorption Fine Structure (NEXAFS) spectroscopy, Fourier transform ion cyclotron resonance mass spectrometry (FT-ICR MS), and optical (absorbance and fluorescence) spectroscopy have been used to probe the chemistry of DOM (Korshin et al., 1998; Wong et al., 2002; Abbt-Braun et al., 2004; Cook, 2004; Del Vecchio and Blough, 2004; McDonald et al., 2004; Christl and Kretzschmar, 2007; Helms et al., 2008; Minor and Stephens, 2008; Schäfer et al., 2009; Berwick et al., 2010; Janot et al., 2010; Matilainen et al., 2011; Zhang et al., 2012). These efforts have conclusively shown that DOM fractions with high molecular weight and hydrophobicity are preferentially removed in water treatment processes 
(Vilgé-Ritter et al., 1999; Leenheer and Croué, 2003; Zhang et al., 2005; Yan et al., 2007).

Despite the extent of these studies, quantitative data correlating water treatment conditions and resultant changes on the intrinsic chemistry of DOM, for instance its proton- and metal-binding capacity and applicable stability constants are limited while such data are highly important for achieving a detailed understanding of DOM interactions with coagulants (Yan et al., 2008; Yan et al., 2013a), absorbents (Janot et al., 2011; Janot et al., 2012) and metal cations (e.g., $\mathrm{Cu}^{2+}, \mathrm{Cd}^{2+}, \mathrm{Fe}^{3+}$ and $\mathrm{Al}^{3+}$ ) (Bai et al., 2008; Marang et al., 2008; Marang et al., 2009; Xiao et al., 2013; Yan et al., 2013a; Yan et al., 2013b; Yan et al., 2013c).

Earlier research has demonstrated that spectrophotometric titrations allow ascertaining interactions of DOM with protons and metal cations (Dryer et al., 2008; Janot et al., 2010; Yan et al., 2013b). In this paper, we expanded this approach and adopted it to characterize DOM originating from critical points in drinking water treatment plants, notably after coagulation and filtration. The protonation properties of DOM present in the inlet (or influent) and in treated water were further quantified via deconvolution that helped discern contributions of discrete bands present. Changes of absorbance-derived parameters determined for DOM in the influent, treated water and selected DOM fractions were interpreted based on the NICA-Donnan Model (Milne et al., 
2001; Ritchie and Perdue, 2003; Dryer et al., 2008; Janot et al., 2010) that helped ascertain properties of low- and high-affinity protonation-active groups in DOM.

\section{Material and Methods}

2.1. Samples. Grab samples of inlet, settled and filtered waters after coagulation from two water treatment plants denoted Plant $A$ and $B$, and located in the Atlantic coast of the United States were collected in 5-gallon cubitainers by the treatment plant personnel and immediately shipped to the research laboratory at the University of Washington. The inlet water for Plant A and $B$ is the same. DOC concentrations in the examined waters were relatively low (Table S1), and prior to fractionation experiments these samples were RO-preconcentrated as described in Supporting Information Section. All chemicals were AR-grade unless otherwise specified. All solutions were prepared using Milli-Q water (18.2 $\mathrm{M} \Omega \mathrm{cm}^{-1}$, Millipore Corp., MA, USA).

2.2. Spectrophotometric titration. Spectrophotometric titration of all five RO isolated DOM samples was carried out as described in previous literature (Dryer et al., 2008; Yan et al., 2013b). During these titrations, the ROpreconcentrated DOM samples were diluted with Milli-Q water to reach a 5.0 $\mathrm{mg} \mathrm{L}^{-1} \mathrm{DOC}$ concentration. 
2.3. DOM Fractionation. DOM fractionation for five RO-preconcentrated water samples was repeated twice using XAD8 and XAD4 resin fractionation scheme (Croue et al., 2000). The DOC in the RO-preconcentrated samples was adjusted to $15 \pm 1 \mathrm{mg} \mathrm{L}^{-1}$, and the sample volume was $520 \mathrm{~mL}$. The first two steps in the fractionation procedure included the separation of the hydrophobic basic (HPOB) and hydrophobic acidic (HPOA) fractions of DOM. The HPOB fraction was retained by XAD8 (or DAX8) resin at $\mathrm{pH} 12$ and eluted by $0.1 \mathrm{M}$ perchloric acid, while the HPOA fraction was retained by XAD8 at $\mathrm{pH}$ 12 and is eluted by $0.1 \mathrm{M}$ sodium hydroxide.

The remainder of DOM that was retained by XAD8 and not eluted by either basic or acidic eluents was assigned to the fraction of hydrophobic neutrals (HPON). Following the separation of hydrophobic (HPO) fractions, similar operations were carried out with XAD4 resin, which retained the transphilic fractions (TPH) of DOM. These included the fractions of transphilic bases (TPHB) retained by XAD4 at $\mathrm{pH} 12$ and eluted by perchloric acid, transphilic acids (TPHA) adsorbed at $\mathrm{pH} 2$ and eluted by sodium hydroxide. The remainder of DOM retained by XAD4 was assigned to the fraction of transphilic neutrals (TPHN). The DOM that was not retained by either XAD8 or XAD4 was classified as hydrophilic DOM fraction (HPI). The effluent of XAD8 or XAD4 resin at $\mathrm{pH} 2$ and $\mathrm{pH} 12$ were collected also to deduce the normalized differential absorbance spectra of HPON and TPHN fraction. 
2.4. Analytical methods. DOC concentrations were determined with a Shimadzu TOC-Vcsh carbon analyzer. Absorbance spectra were recorded using a Perkin-Elmer Lambda 18 UV/Vis spectrophotometer with a $5 \mathrm{~cm}$ cell. All absorbance spectra were measured at $\mathrm{pH} 2.0$, except in the spectrophotometric titration experiment where $\mathrm{pH}$ was varied as needed.

\section{Results and Discussion}

\subsection{DOMs fractionated by RA in water treatment processes}

DOM fractionation showed that the examined samples had similar compositions (Table 1). The most notable difference in their fractional compositions was a relatively larger contribution of the HPOA fraction in inlet water DOM. The percentages of HPON fractions were relatively high, and the contributions of all hydrophobic (HPO) fractions were on the average close to $60 \%$.

The contributions of transphilic (TPH) and HPI fractions were comparable for all five examined samples, with the percentages of the HPI fraction (typically close to $10 \%$ ) being low. This also possibly indicates that a fraction of hydrophilic organic molecules associated with the HPI fraction in the original samples may have been lost during RO isolation. The average recovery of DOC during RO operations was close to $90 \%$ (Table S1).

\section{Table 1}


3.2. Changes of spectroscopic properties of DOMs in water treatment processes

The absorbances of DOM decreased gradually after settling and filtering with

142

DOC removal. To compare the changes of spectroscopic properties of DOMs caused by settling and filtering, the absorbance of DOMs in inlet, settled and filtered water from the examined treatment plants were normalized by their DOC concentrations (Figure S2). The DOC-normalized absorbance of DOMs decreased also in settled and filtered water. In all cases, the absorbance intensity decreased exponentially with the observation wavelength, as observed in prior studies (Twardowski et al., 2004; Yan et al., 2013b; Yan et al., 2013c). To ascertain changes of DOM absorbance caused by the treatment of the inlet water in more detail, the differential DOC-normalized spectra of treated waters were calculated using the spectrum of the inlet water as a reference (Eq 1):

$$
\Delta A_{\lambda}=\frac{A_{\lambda, i}}{D O C}-\frac{A_{\lambda, r e f}}{D O C}
$$

where $A_{\lambda, i}$ and $A_{\lambda, \text { ref }}$ are absorbances of the treated and inlet water, respectively (Dryer et al., 2008). Results of these calculations (Figure 1) demonstrate that the intensity of the differential spectra of both settled waters decrease with the wavelength while the differential spectra for filtered waters have a peak with maximum located near to wavelength $270 \mathrm{~nm}$. The degree of 
change of absorbance was more obvious for Plant A than Plant B, which was consistent with the efficiency of DOM removal and indicates that the DOC with higher absorbance is more removable (Table S1).

\section{Figure 1}

The differential DOC-normalized spectra were processed to determine the presence of constituting bands (Yan et al., 2013b). Results of this processing (Figure 2) demonstrate that the fitting of the experimental spectra with six Gaussian distribution band components resulted in a very close fit $\left(R^{2}>0.95\right)$. The constituting bands operationally referred to as A0 $(210 \mathrm{~nm}), \mathrm{A} 1(236 \mathrm{~nm})$, A2 $(270 \mathrm{~nm}), \mathrm{A} 3(313 \mathrm{~nm}), \mathrm{A} 4(374 \mathrm{~nm})$ and A5 (523 nm). The positions of bands $A 1, A 2, A 3$ and $A 4$ are consistent with the results in our prior studies (Yan et al., 2013b). Figure 2 indicates the difference between the differential DOC-normalized spectra calculated for settled and filtered water was primarily associated with the high contribution of Band $A 2$ while that of Band $A 1$ is low.

\section{Figure 2}

To examine differences in the properties of DOM chromophores present in different fractions, the differential spectra were normalized by their intensity at $220 \mathrm{~nm}$ (Yan et al, 2012). The normalized differential absorbance spectra of each fraction calculated using the data for the sample before fractionation were calculated using Eq 2. 


$$
R \Delta A_{\lambda}=\frac{A_{\lambda, i}}{A_{220, i}}-\frac{A_{\lambda, \text { ref }}}{A_{220, r e f}}
$$

180 In this expression, $R \Delta A_{\lambda}$ is the normalized differential absorbance at

181 wavelength $\lambda . A_{\lambda, i}$ and $A_{\lambda, r e f}$ are absorbance spectra at a particular 182 wavelength measured for selected fraction and DOM before fractionation, 183 respectively.

\section{Figure 3}

The normalized differential spectra could be fitted the same Gaussian 186 components which were found in the differential spectra of DOM in previous study $\left(R^{2}>0.95\right)$ (Figure 4) but the contributions of these bands were different (Yan et al., 2013b). HPOB fraction had the highest intensity of Band A1 while 189 the HPOA fraction had significantly higher contributions of bands A1 and A2, 190 but relatively lower contribution of band A4; TPHA had higher contribution of Band A1 and A2, especially for A2, and lowest contribution of Band A3; TPHB was of negative value for all the bands except $\mathrm{A} 1$ and the contribution of $\mathrm{A} 3$ was very significant; TPHN had high contributions of Band A1 and Band A2, 194 and relatively lower contributions of A3 and A4. HPI had significant negative 195 value of all the bands.

Based on the comparison of the results shown in Figure 1 and 2, it could be 197 also concluded that settling and filtering appears to remove efficiently the 
fractions such as HPOB, HPOA, HPON, and TPHA, with the filtration step associated with further removal of the HPOA and TPHA fractions.

\section{Figure 4.}

\subsection{Effects of $\mathrm{pH}$ variation on DOM absorbance spectra.}

To ascertain changes of DOM acid-base property induced by water treatment, the absorbances of all DOM samples were measured at $\mathrm{pH}$ values ranging from 2.5 to 10.0 . The corresponding zero-order and $\mathrm{pH}$-differential spectra calculated using Eq 1 as described in prior studies (Dryer et al. 2008; Yan et al. 2013b) from the reference $\mathrm{pH}$ value of 2.5 are shown in Figure $\mathrm{S} 4$ and Figure 5 , respectively.

The intensities of differential absorbances differ significantly between the five samples but major trends discerned from the figures were similar. All $\mathrm{pH}$-differential spectra had peaks located at approximately 245,280 and 370 $\mathrm{nm}$ and for all five DOM samples, the spectra could be deconvoluted using the six Gaussian components $\left(R^{2}>0.995\right)$ as shown in Figure 6.

\section{Figure 5.}

\section{Figure 6.}

Prior research has shown that in the system of Gaussian components used to deconvolute the differential spectra of DOM, bands A4 and A5 are most 
characteristic for humic substances (Yan et al., 2013b) because they appear to reflect contributions of the inter-chromophore interactions in humic species (Del Vecchio and Blough, 2004; Ma et al., 2010). The relatively low intensity of Band A4 necessitates that the log-transformed and differential spectra be calculated using the log-transformed data using Eq 3:

$$
D \ln A_{\lambda}=\ln A_{\lambda, i}-\ln A_{\lambda, \text { ref }}
$$

Results of data processing using the above equation are shown in Figure 7. As discussed in previous literature (Yan et al., 2013 a, 2013c), the intensity of the differential log-transformed absorbance at wavelength $350 \mathrm{~nm}$ is reflective of changes of Band A4. Accordingly, DInA $A_{350}$ values for all five isolated DOMs in the entire ranges of $\mathrm{pH}$ used in the experiments were calculated and presented in Figure 8.

\section{Figure 7.}

\section{Figure 8.}

\subsection{Modeling proton binding onto DOMs using NICA-Donnan Model}

Major trends in the behavior of $\mathrm{DlnA}_{350} \mathrm{vs}$. $\mathrm{pH}$ for were similar for the five examined waters. To quantify the modification of DOM in water treatment processes, the $\mathrm{DlnA}_{350}$ vs. $\mathrm{pH}$ datasets were interpreted based on the NICA-Donnan theory as described in previous studies (Milne et al., 2001; Ritchie and Perdue, 2003; Dryer et al., 2008; Janot et al., 2010): 


$$
\begin{gathered}
D \ln A_{p H}=\left[\frac{D \ln A_{L A S}}{1+\left(\tilde{K}_{L A S}\left[H^{+}\right]\right)^{m_{L A S}}}+\frac{D \ln A_{H A S}}{1+\left(\tilde{K}_{H A S}\left[H^{+}\right]\right)^{m_{H A S}}}\right]- \\
-\left[\frac{D \ln A_{L A S}}{1+\left(\tilde{K}_{L A S}^{\left.\left[H^{+}\right]_{r e f}\right)^{m_{L A S}}}+\frac{D \ln A_{H A S}}{1+\left(\tilde{K}_{H A S}\left[H^{+}\right]_{r e f}\right)^{m_{H A S}}}\right]}\right.
\end{gathered}
$$

In the above formula, $D \ln A_{L A S}$ and $D \ln A_{H A S}$ correspond to the maximum

239 change of absorbance associated with the deprotonation of the low-affinity 240 protonation-active groups (LAS, mostly carboxylic) and high-affinity 241 protonation-active groups (HAS, mostly phenolic), respectively, where $242 D \ln A_{L A S}$ and $D \ln A_{H A S}$ refer to DInA values at wavelength $350 \mathrm{~nm}$. It needs to 243 be noted that $D \ln A_{L A S}$ and $D \ln A_{H A S}$ are parameters independent of $D O C$ 244 concentration, and they reflect the amount of protonation-active groups in per $245 \mathrm{mg} \mathrm{L}-1$ DOM. $\tilde{K}_{L A S}$ and $\tilde{K}_{H A S}$ are the median values of the protons affinity 246 distributions for these groups, $m_{L A S}$ and $m_{H A S}$ define the width of these 247 distributions and are measures of the heterogeneity of DOM (Milne et al., 2001; 248 Dryer et al., 2008; Yan et al., 2014).

249 The behavior of $\mathrm{DInA}_{350}$ values for all five DOMs vs. $\mathrm{pH}$ and their fitting are 250 shown in Figure 8 and Table 2. The data show that excellent agreement can 251 be reached between the $\operatorname{Dln} A_{350}$ data for all samples and the predictions made based on the revised NICA-Donnan Model $\left(R^{2}>0.99\right)$. 
254 The $\tilde{K}_{L A S}$ and $\tilde{K}_{H A S}$ values for all five DOMs determined in this study are 255 similar, and are in the range of those reported in the literature (Milne et al., 256 2001; Ritchie and Perdue, 2003). $\tilde{K}_{L A S}$ values increase slightly after 257 settlement and filtration in Plant A water plantwhile $\tilde{K}_{H A S}$ values for waters of 258 both plants decreased after treatment and it is lower in DOM after settled 259 waters than that in after filtered water.

260 The DInA $\angle A S$ and DInA $A_{H A S}$ values increased in the treated waters, especially for 261 Plant $A$. This demonstrates that the normalized amounts of the LAS and HAS 262 groups in DOM remaining in water after settling and filtering steps is more abundant than in Inlet water, and these groups are more sensitive to variations of $\mathrm{pH}$. This is consistent with hydrophobic DOM being more available to be removed in water treatment. The ratio of $D \ln A_{L A S} / D \ln A_{H A S}$ also increased in treated waters indicating that DOM with high-affinity groups (mostly phenolic-like groups) are preferentially removed during water treatment.

The relative changes of the concentrations of LAS and HAS groups in DOM determined based on NICA-Donnan modeling was compared with the results of DOM fractionation. Results of this comparison are shown in Figure 9 and S5.

271 These indicate that the relative contributions of the LAS and HAS groups 272 increase linearly with the percentage of $\mathrm{HPI}$ fraction $\left(R^{2} 0.91\right.$, excluding the 273 data for filtered water from Plant B which appear to be an outlier) in Figure S5(a)). The concentrations of HAS decreased with the total percentages of 
HPOB and TPHB fractions (Figure S5(b), $R^{2}$ 0.92, excluding the data for the settled water produced by Plant B)). The contribution of HAS in the total protonation active groups increased with the total percentages of HPOA, TPHA and $\mathrm{HI}$ fractions (Figure 9, $\mathrm{R}^{2}=0.95$ ).

\section{Figure 9}

\section{Conclusions}

This study used absorbance spectroscopy to quantify changes of DOM deprotonation-protonation properties in drinking water produced by two treatment plants.

(1) The data demonstrate that properties of the chromophores in DOM present in inlet, settled and filtered waters and their fractions can be quantified via numeric deconvolution of the relevant differential spectra. This approach indicated the presence of six well-resolved Gaussian bands A0 $(210 \mathrm{~nm})$, A1 (236 nm), A2 (270 nm), A3 (313 nm), A4 (374 nm) and A5 (523 nm) that comprise the spectra in all examined cases.

(2) The emergence of these bands is associated with the carboxylic and phenolic functionalities. DOM fractions with high contributions of band $\mathrm{A} 1$ and $A 2$, e.g. the HPOB, HPOA, HPON, and TPHA fractions are more easily removed by settling and filtering. Filtration is more efficient than 
settle in removing DOM with high contribution of band $A 2$ on verse and the band $\mathrm{A} 1$ is low, e.g. the HPOA and TPHA fractions.

(3) The differential absorbance of DOM presented at each water treatment stage caused by deprotonation could reflect the amount of protonation-active chromophores in DOM. When combined with the NICA-Donnan model, the constants describing DOM/proton equilibria could be calculated based on the observed changes of the selected parameter $\mathrm{DInA}_{350} \mathrm{vs} \mathrm{pH}$.

(4) The normalized content of low- and high-affinity protonation-active groups (LAS and HAS) in DOM remaining in the treated water after settling and filtering steps was found to be higher than that in Inlet water. The observed changes of the LAS and HAS were generally consistent with the polarity characteristics of DOMs fractionated by XAD8/XAD4 RA.

\section{Acknowledgements}

This study was partially supported by the Cadmus (grant 069-UW-1). Further work on and interpretation of the results was partially supported by China NSF (No. 51578007). The views represented in this publication do not necessarily represent those of the funding agencies. 
313

314

315

\section{Reference}

Abbt-Braun, G., Lankes, U., Frimmel, F., 2004. Structural characterization of aquatic humic substances - The need for a multiple method approach. Aquat Sci 66, 151-170.

Bai, Y.C., Wu, F.C., Liu, C.Q., Li, W., Guo, J.Y., Fu, P.Q., Xing, B.S., Zheng, J., 2008. Ultraviolet absorbance titration for determining stability constants of humic substances with $\mathrm{Cu}(\mathrm{II})$ and $\mathrm{Hg}(\mathrm{II})$. Anal Chim Acta 616, 115-121.

Berwick, L., Greenwood, P.F., Smernik, R.J., 2010. The use of MSSV pyrolysis to assist the molecular characterisation of aquatic natural organic matter. Water Res 44, 3039-3054.

Christl, I., Kretzschmar, R., 2007. C-1s NEXAFS spectroscopy reveals chemical fractionation of humic acid by cation-induced coagulation. Environ Sci Technol 41, 1915-1920.

Cook, R., 2004. Coupling NMR to NOM. Anal Bioanal Chem 378, 1484-1503. Croue, J.P., Benedetti, M.F., Violleau, D., Leenheer, J.A., 2003. Characterization and copper binding of humic and nonhumic organic matter isolated from the South Platte River: Evidence for the presence of nitrogenous binding site. Environ Sci Technol 37, 328-336.

Croue, J.P., Korshin, G.V., Benjamin, M.M., Foundation, A.R., 2000. Characterization of Natural Organic Matter in Drinking Water. AWWA Research Foundation and American Water Works Association.

Del Vecchio, R., Blough, N.V., 2004. On the origin of the optical properties of 
humic substances. Environ Sci Technol 38, 3885-3891.

Ding, G.Y., Zhang, X.R., 2009. A picture of polar iodinated disinfection byproducts in drinking water by (UPLC/)ESI-tqMS. Environ Sci Technol 43, 9287-9293.

Dryer, D.J., Korshin, G.V., Fabbricino, M., 2008. In situ examination of the protonation behavior of fulvic acids using differential absorbance spectroscopy. Environ Sci Technol 42, 6644-6649.

Helms, J.R., Stubbins, A., Ritchie, J.D., Minor, E.C., Kieber, D.J., Mopper, K., 2008. Absorption spectral slopes and slope ratios as indicators of molecular weight, source, and photobleaching of chromophoric dissolved organic matter. Limnol Oceanogr 53, 955-969.

Janot, N., Benedetti, M.F., Reiller, P.E., 2011. Colloidal alpha-Al2O3, europium(III) and humic substances interactions: A macroscopic and spectroscopic study. Environ Sci Technol 45, 3224-3230.

Janot, N., Reiller, P.E., Korshin, G.V., Benedetti, M.F., 2010. Using spectrophotometric titrations to characterize humic acid reactivity at environmental concentrations. Environ Sci Technol 44, 6782-6788.

Janot, N., Reiller, P.E., Zheng, X., Croue, J.P., Benedetti, M.F., 2012. Characterization of humic acid reactivity modifications due to adsorption onto alpha-Al2O3. Water Res 46, 731-740.

Korshin, G.V., Frenkel, A.I., Stern, E.A., 1998. EXAFS study of the inner shell structure in copper(II) complexes with humic substances. Environ Sci Technol 
32, 2699-2705.

Leenheer, J.A., Croué, J.-P., 2003. Characterizing aquatic dissolved organic matter. Environ Sci Technol 37, 18A-26A.

Liu, G., Zhang, X., Talley, J.W., 2007. Effect of copper (II) on natural organic matter removal during drinking water coagulation using aluminum-based coagulants. Water Environ Res 79, 593-599.

Ma, J.H., Del Vecchio, R., Golanoski, K.S., Boyle, E.S., Blough, N.V., 2010. Optical Properties of Humic Substances and CDOM: Effects of Borohydride Reduction. Environ Sci Technol 44, 5395-5402.

Marang, L., Eidner, S., Kumke, M.U., Benedetti, M.F., Reiller, P.E., 2009. Spectroscopic characterization of the competitive binding of $\mathrm{Eu}(\mathrm{III}), \mathrm{Ca}(\mathrm{II})$, and $\mathrm{Cu}$ (II) to a sedimentary originated humic acid. Chem Geol 264, 154-161.

Marang, L., Reiller, P.E., Eidner, S., Kumke, M.U., Benedetti, M.F., 2008. Combining spectroscopic and potentiometric approaches to characterize competitive binding to humic substances. Environ Sci Technol 42, 5094-5098.

Matilainen, A., Gjessing, E.T., Lahtinen, T., Hed, L., Bhatnagar, A., Sillanpaa, M., 2011. An overview of the methods used in the characterisation of natural organic matter (NOM) in relation to drinking water treatment. Chemosphere 83, 1431-1442.

McDonald, S., Bishop, A.G., Prenzler, P.D., Robards, K., 2004. Analytical chemistry of freshwater humic substances. Anal Chim Acta 527, 105-124.

Milne, C.J., Kinniburgh, D.G., Tipping, E., 2001. Generic NICA-Donnan model 
parameters for proton binding by humic substances. Environ Sci Technol 35, 2049-2059.

Minor, E., Stephens, B., 2008. Dissolved organic matter characteristics within the Lake Superior watershed. Org Geochem 39, 1489-1501.

Muller, M.B., Schmitt, D., Frimmel, F.H., 2000. Fractionation of natural organic matter by size exclusion chromatography - Properties and stability of fractions.

\section{Environ Sci Technol 34, 4867-4872.}

Park, J.H., 2009. Spectroscopic characterization of dissolved organic matter and its interactions with metals in surface waters using size exclusion chromatography. Chemosphere 77, 485-494.

Richardson, S.D., Plewa, M.J., Wagner, E.D., Schoeny, R., Demarini, D.M., 2007. Occurrence, genotoxicity, and carcinogenicity of regulated and emerging disinfection by-products in drinking water: a review and roadmap for research. Mutat Res 636, 178-242.

Richardson, S.D., Ternes, T.A., 2011. Water analysis: emerging contaminants and current issues. Anal Chem 83, 4614-4648.

Ritchie, J.D., Perdue, E.M., 2003. Proton-binding study of standard and reference fulvic acids, humic acids, and natural organic matter. Geochim Cosmochim Ac 67, 85-96.

Schäfer, T., Michel, P., Claret, F., Beetz, T., Wirick, S., Jacobsen, C., 2009. Radiation sensitivity of natural organic matter: Clay mineral association effects in the Callovo-Oxfordian argillite. J Electron Spectrosc 170, 49-56. 
401

402

403

404

405

406

407

Schafer, A.I., Mauch, R., Waite, T.D., Fane, A.G., 2002. Charge effects in the fractionation of natural organics using ultrafiltration. Environ Sci Technol 36, 2572-2580.

Thurman, E.M., Malcolm, R.L., 1981. Preparative isolation of aquatic humic substances. Environ Sci Technol 15, 463-466.

Twardowski, M.S., Boss, E., Sullivan, J.M., Donaghay, P.L., 2004. Modeling the spectral shape of absorption by chromophoric dissolved organic matter. Mar Chem 89, 69-88.

Vilgé-Ritter, A., Masion, A., Boulangé, T., Rybacki, D., Bottero, J.-Y., 1999. Removal of natural organic matter by coagulation-flocculation: $A$ pyrolysis-GC-MS study. Environ Sci Technol 33, 3027-3032.

Wong, S., Hanna, J.V., King, S., Carroll, T.J., Eldridge, R.J., Dixon, D.R., Bolto, B.A., Hesse, S., Abbt-Braun, G., Frimmel, F.H., 2002. Fractionation of natural organic matter in drinking water and characterization by C-13 cross-polarization magic-angle spinning NMR spectroscopy and size exclusion chromatography. Environ Sci Technol 36, 3497-3503.

Xiao, Y.H., Sara-Aho, T., Hartikainen, H., Vahatalo, A.V., 2013. Contribution of ferric iron to light absorption by chromophoric dissolved organic matter. Limnol Oceanogr 58, 653-662.

Yan, M.-q., Wang, D.-s., SHI, B.-y., WEI, Q.-s., QU, J.-h., Tang, H.-x., 2007. Transformations of particles, metal elements and natural organic matter in different water treatment processes. J Environ Sci 19, 271-277. 
423

424

425

426

427

428

429

430

431

432

433

434

435

436

437

Yan, M., Benedetti, M.F., Korshin, G.V., 2013a. Study of iron and aluminum binding to Suwannee River fulvic acid using absorbance and fluorescence spectroscopy: Comparison of data interpretation based on NICA-Donnan and Stockholm humic models. Water Res 47, 5439-5446.

Yan, M., Dryer, D., Korshin, G.V., Benedetti, M.F., 2013b. In situ study of binding of copper by fulvic acid: Comparison of differential absorbance data and model predictions. Water Res 47, 588-596.

Yan, M., Korshin, G.V., Claret, F., Croué, J.-P., Fabbricino, M., Gallard, H., Schäfer, T., Benedetti, M.F., 2014. Effects of charging on the chromophores of dissolved organic matter from the Rio Negro basin. Water Res 59, 154-164.

Yan, M., Wang, D., Korshin, G.V., Benedetti, M.F., 2013c. Quantifying metal ions binding onto dissolved organic matter using log-transformed absorbance spectra. Water Res 47, 2603-2611.

Yan, M., Wang, D., Ni, J., Qu, J., Chow, C.W.K., Liu, H., 2008. Mechanism of natural organic matter removal by polyaluminum chloride: Effect of coagulant particle size and hydrolysis kinetics. Water Res 42, 3361-3370.

Zhang, H., Zhang, Y., Shi, Q., Ren, S., Yu, J., Ji, F., Luo, W., Yang, M., 2012. Characterization of low molecular weight dissolved natural organic matter along the treatment trait of a waterworks using Fourier transform ion cyclotron resonance mass spectrometry. Water Res 46, 5197-5204.

Zhang, X., Minear, R.A., Barrett, S.E., 2005. Characterization of high molecular weight disinfection byproducts from chlorination of humic 
445 substances with/without coagulation pretreatment using UF-SEC-ESI-MS/MS.

446 Environ Sci Technol 39, 963-972.

447

448 
450 Table 1 Contributions of hydrophobic, transphilic and hydrophilic fractions in 451 the concentrations of dissolved organic carbon in RO isolates of influent (inlet), 452 Plant A settled (AS) and filtered (AF), and Plant B settled (BS) and filtered (BF) 453 water.

454 Table 2 NICA modeling parameters for protonation-active groups in DOMs 455 determined by DInA350 in spectrophotometric titrations. DOMs in RO isolates 456 of influent (inlet), Plant A settled (AS) and filtered (AF), and Plant B settled (BS) 457 and filtered (BF) water. Figure 1 DOC-normalized differential absorbances of DOM in RO isolates of Plant A settled (AS) and filtered (AF), and Plant B settled (BS) and filtered (BF) waters vs. the absorbance spectrum of the influent (inlet water).

Figure 2 Gaussian band fitting of the DOC-normalized differential absorbance of DOM in RO isolates of Plant A settled (AS, a) and filtered (AF, b), and Plant $B$ settled (BS, c) and filtered water (BF, d). Absorbance spectrum of the 464 influent (inlet water) was used as the reference.

Figure 3. Normalized differential absorbance of hydrophobic, transphilic and hydrophilic fractions in DOM in RO isolates of influent (inlet) water. Normalized by absorbance at wavelength $220 \mathrm{~nm}$, reference to DOM before fractionation. The normalized differential absorbance spectra of HPON and TPHN fraction 
469 were deduced by the normalized differential absorbance spectra of the effluent 470 of $\mathrm{XAD} 8$ or $\mathrm{XAD} 4$ resin at $\mathrm{pH} 2$ and $\mathrm{pH} 12$, respectively.

471 Figure 4. Gaussian band fitting of the normalized differential absorbance by 472 intensity at $220 \mathrm{~nm}$ and DOC concentration of DOM fractions in water 473 treatment processes referenced to the inlet waters.

474 Figure 5. pH-differential absorbance spectra of normalized differential 475 absorbance of DOMs in RO isolates of DOM in the influent (inlet, a), Plant A 476 settled (AS, b) and filtered (AF, c), and Plant B settled (BS, d) and filtered (BF, 477 e) water. Reference pH value 2.5 . 
Figure 6. Gaussian band fitting of the differential DOM spectra in the influent (inlet, a), Plant A settled (AS, b) and filtered (AF, c), and Plant B settled (BS, d) and filtered $(\mathrm{BF}, \mathrm{e})$ water at $\mathrm{pH}$ about 7.0 referred to $\mathrm{pH} 2.5$.

Figure 7. Log-transformed differential absorbance spectra of normalized differential absorbance of DOM in the influent (inlet, a), Plant A settled (AS, b) and filtered (AF, c), and Plant $B$ settled (BS, d) and filtered (BF, e) water. Reference $\mathrm{pH}$ value 2.5 .

Figure 8. Comparison of effects of $\mathrm{pH}$ on the differential log-transformed absorbance at wavelength $350 \mathrm{~nm}$ and their NICA-based fitting for DOMs in RO isolates of influent (inlet), Plant A settled (AS) and filtered (AF ) and Plant B settled (BS) and filtered (BF) water.

Figure 9. Correlation between the percentage of HAS in total protonation-active groups vs. HPOA, THPA and HPI. DOMs in RO isolates of influent (inlet), Plant A settled (AS) and filtered (AF), and Plant B settled (BS) and filtered (BF) water. 
493 Table 1 Contributions of hydrophobic, transphilic and hydrophilic fractions in 494 the concentrations of dissolved organic carbon in RO isolates of influent (inlet), 495 Plant A settled (AS) and filtered (AF), and Plant B settled (BS) and filtered (BF) 496 water.

\begin{tabular}{|lllllll|}
\hline DOM fraction & Abbreviation & Inlet & AS & AF & BS & BF \\
Hydrophobic bases & HPOB & $10 \%$ & $9 \%$ & $8 \%$ & $8 \%$ & $9 \%$ \\
Hydrophobic acids & HPOA & $24 \%$ & $15 \%$ & $14 \%$ & $17 \%$ & $14 \%$ \\
Hydrophobic neutrals & HPON & $39 \%$ & $48 \%$ & $48 \%$ & $46 \%$ & $44 \%$ \\
Transphilic bases & TPHB & $3 \%$ & $3 \%$ & $2 \%$ & $1 \%$ & $2 \%$ \\
Transphilic acids & TPHA & $3 \%$ & $3 \%$ & $4 \%$ & $3 \%$ & $4 \%$ \\
Transphilic neutrals & TPHN & $12 \%$ & $13 \%$ & $13 \%$ & $15 \%$ & $14 \%$ \\
Hydrophilic fraction & HPI & $9 \%$ & $10 \%$ & $11 \%$ & $10 \%$ & $13 \%$ \\
\hline
\end{tabular}


Table 2 NICA modeling parameters for protonation-active groups in DOMs 498 determined by DInA350 in spectrophotometric titrations. DOMs in RO isolates 499 of influent (inlet), Plant A settled (AS) and filtered (AF), and Plant B settled (BS) 500 and filtered (BF) water.

\begin{tabular}{|l|r|r|r|r|r|}
\hline & Inlet & \multicolumn{1}{l|}{ AS } & \multicolumn{1}{l|}{ AF } & \multicolumn{1}{l|}{ BS } & \multicolumn{1}{l|}{ BF } \\
\hline$\tilde{K}_{L A S}$ & 2.68 & 2.80 & 2.88 & 2.64 & 2.64 \\
\hline$\tilde{K}_{\text {HAS }}$ & 6.58 & 6.07 & 6.22 & 6.10 & 6.30 \\
\hline $\operatorname{DInA}_{L A S}$ & 0.214 & 0.371 & 0.472 & 0.303 & 0.334 \\
\hline$D_{\ln A_{H A S}}$ & 0.238 & 0.272 & 0.327 & 0.241 & 0.278 \\
\hline$m_{L A S}$ & 0.99 & 0.71 & 0.73 & 0.75 & 0.85 \\
\hline$m_{H A S}$ & 0.67 & 0.68 & 0.73 & 0.84 & 0.64 \\
\hline
\end{tabular}




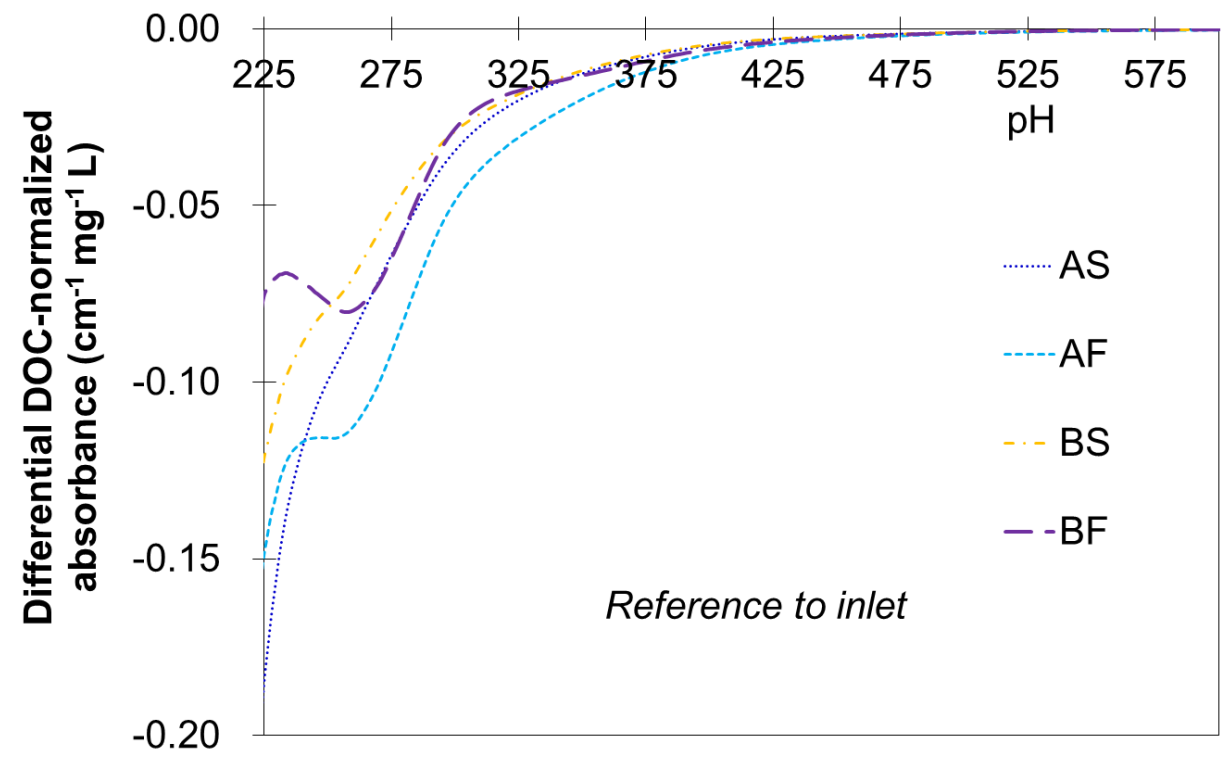

Wavelength (nm)

502 Figure 1 DOC-normalized differential absorbances of DOM in RO isolates of 503 Plant A settled (AS) and filtered (AF), and Plant B settled (BS) and filtered (BF) waters vs. the absorbance spectrum of influent (inlet water) 


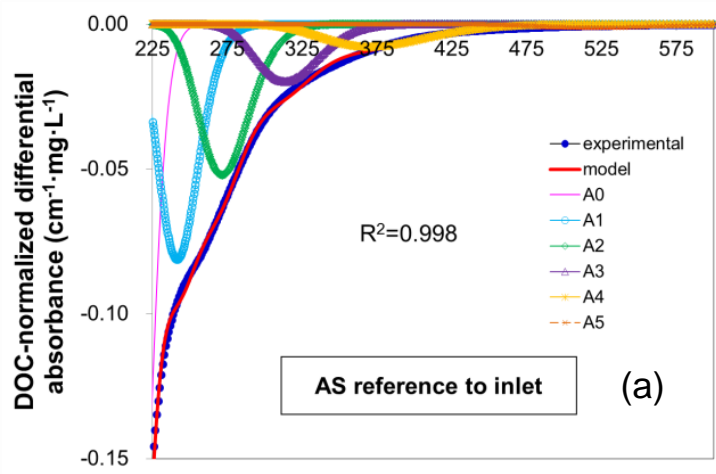

Wavelength (nm)

505

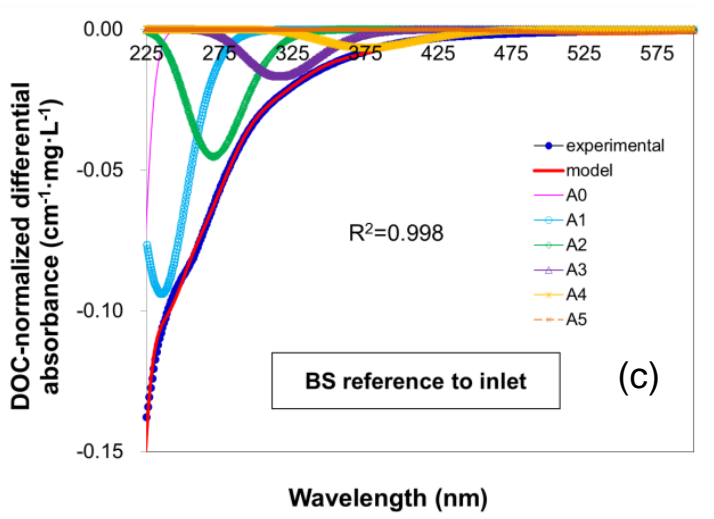

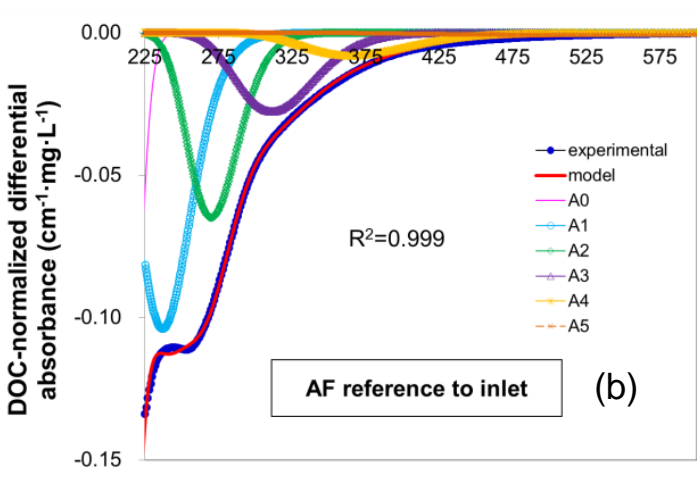

Wavelength (nm)

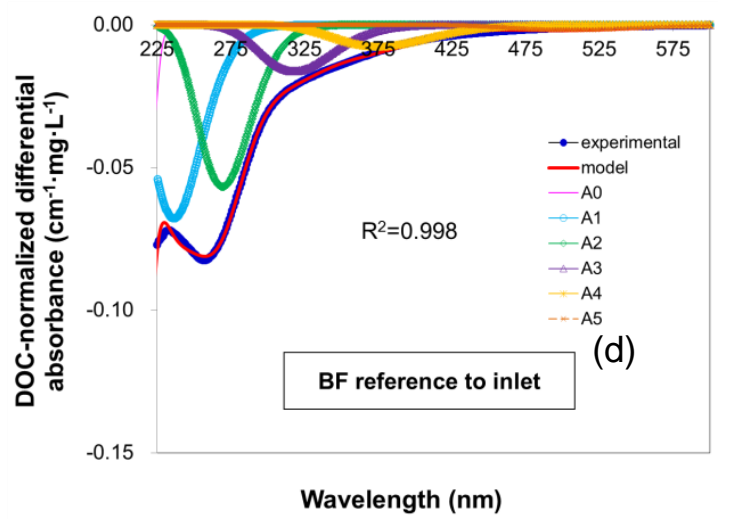

Figure 2 Gaussian band fitting of the DOC-normalized differential absorbance

$B$ settled $(B S, c)$ and filtered water $(B F, d)$. Absorbance spectrum of the 510 influent (inlet) 


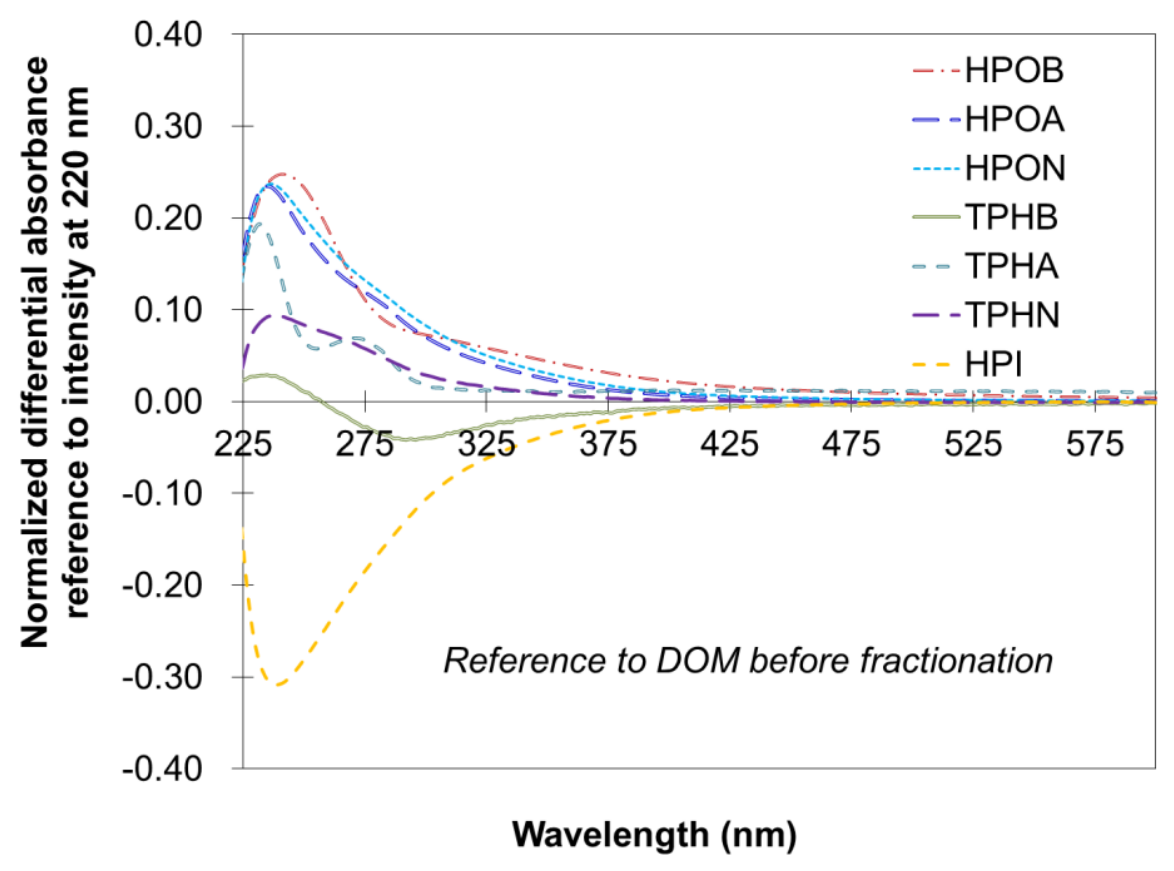

512 Figure 3. Normalized differential absorbance of hydrophobic, transphilic and 513 hydrophilic fractions in DOM in RO isolates of influent (inlet) water. Normalized 514 by absorbance at wavelength $220 \mathrm{~nm}$, reference to DOM before fractionation. 515 The normalized differential absorbance spectra of HPON and TPHN fraction 516 were deduced by the normalized differential absorbance spectra of the effluent 517 of XAD8 or XAD4 resin at $\mathrm{pH} 2$ and $\mathrm{pH} 12$, respectively. 

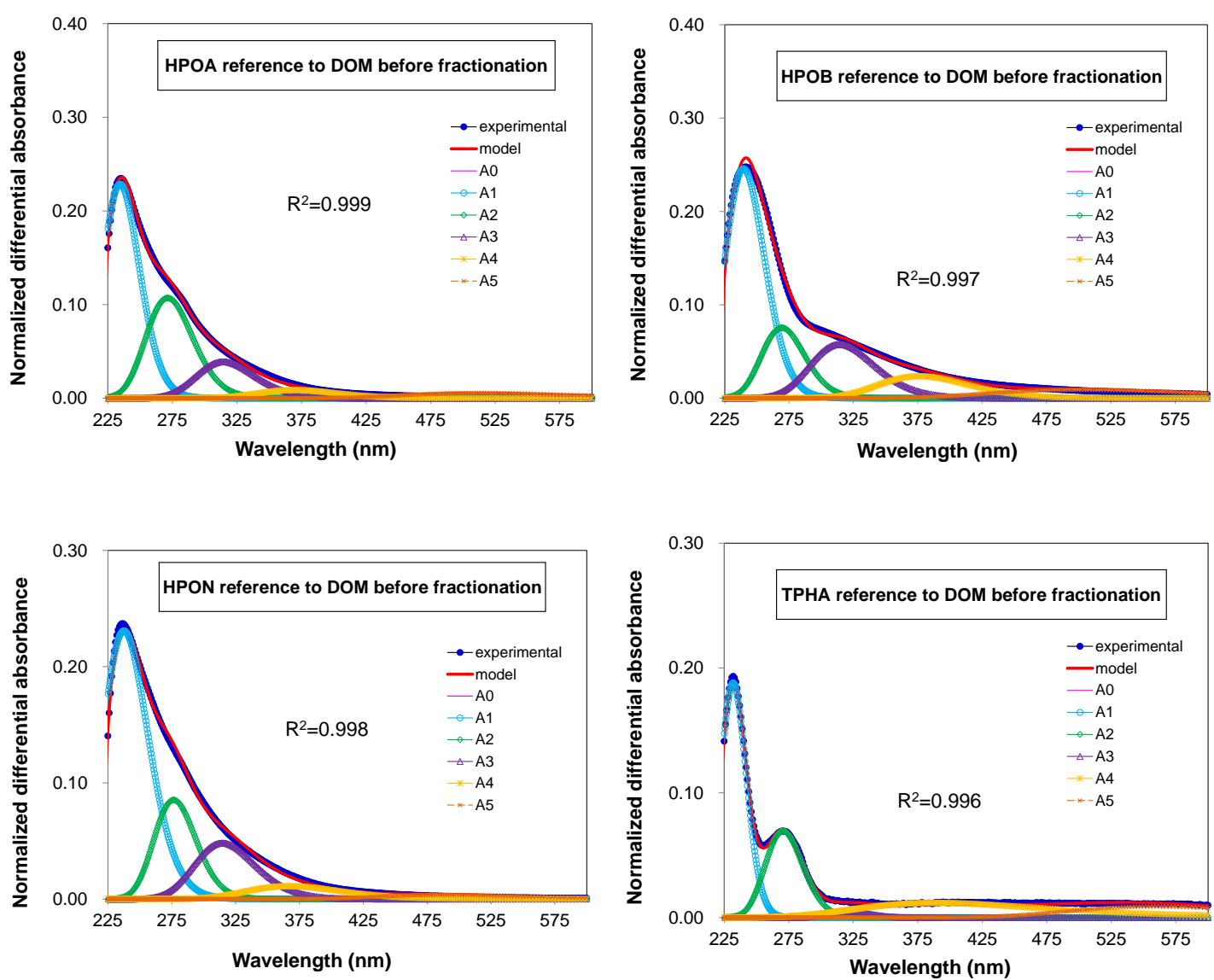

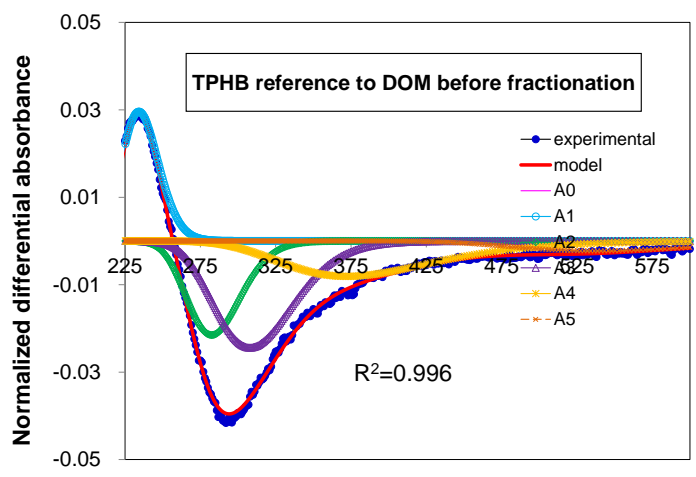

Wavelength $(\mathrm{nm})$

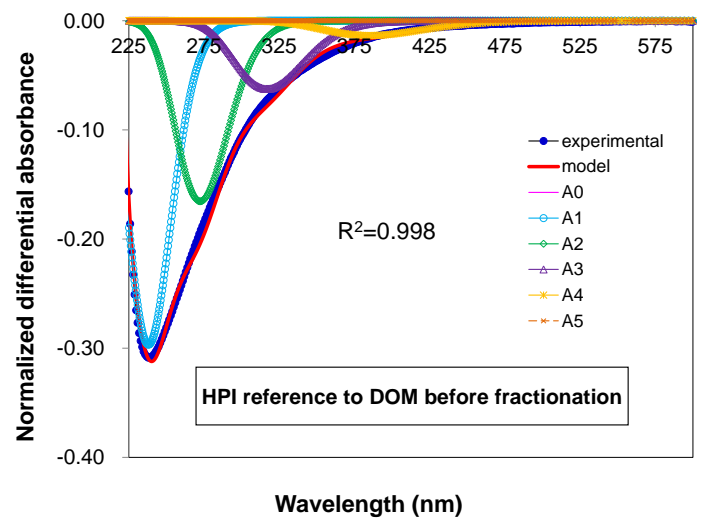

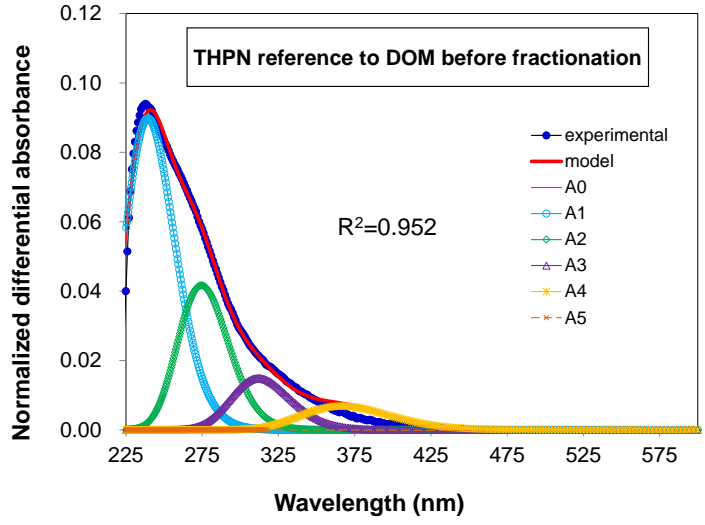

521 
522 Figure 4. Gaussian band fitting of the normalized differential absorbance by

523 intensity at $220 \mathrm{~nm}$ and DOC concentration of DOM fractions in water 524 treatment processes referenced to the inlet waters. 

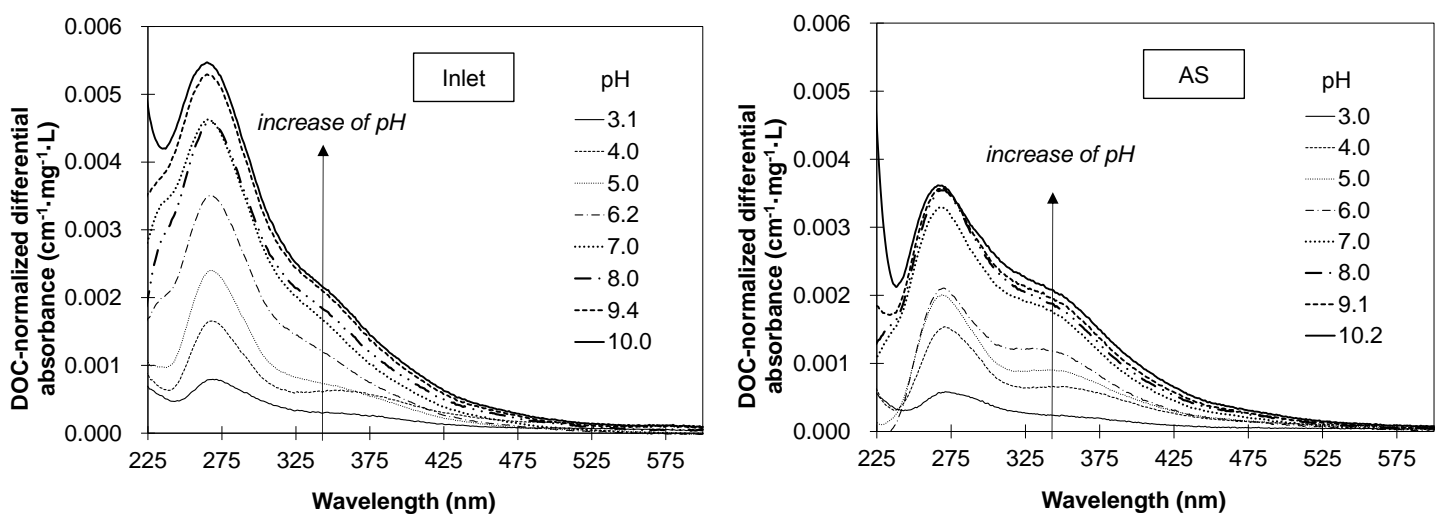

525
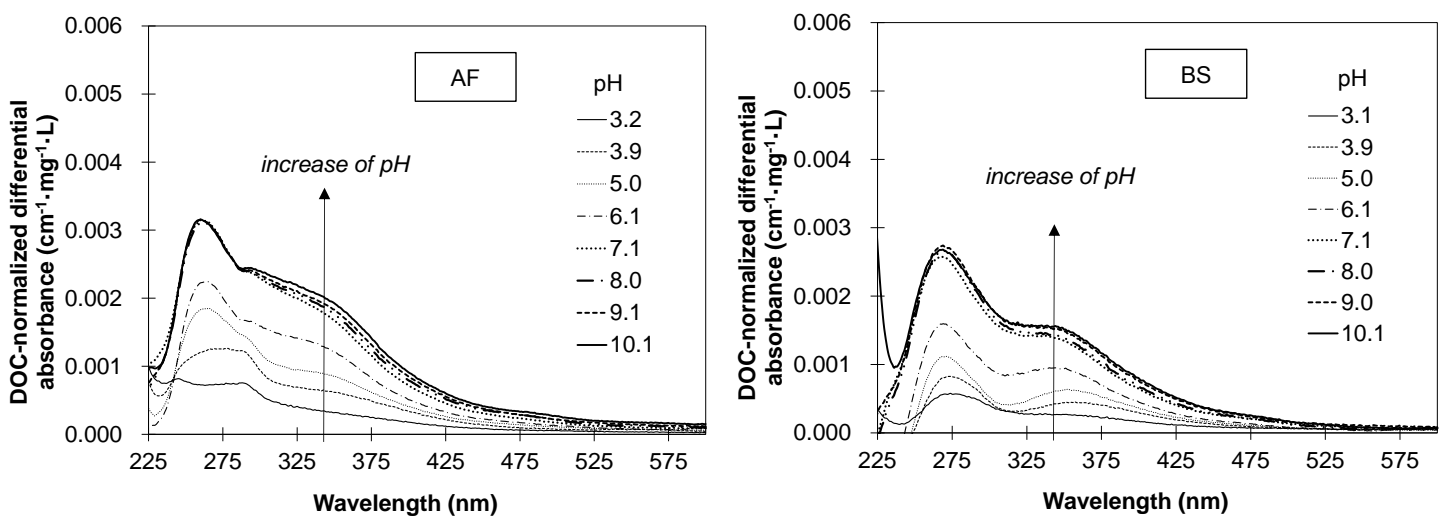

526

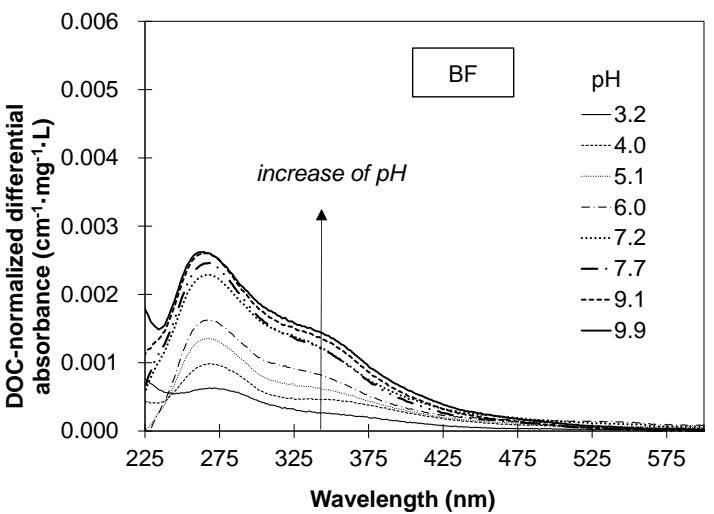

527

528 Figure 5. pH-differential absorbance spectra of normalized differential 529 absorbance of DOMs in RO isolates of influent (inlet, a), Plant A settled (AS, b) and filtered (AF, c), and Plant $B$ settled (BS, d) and filtered (BF, e) water. Reference $\mathrm{pH}$ value 2.5 . 

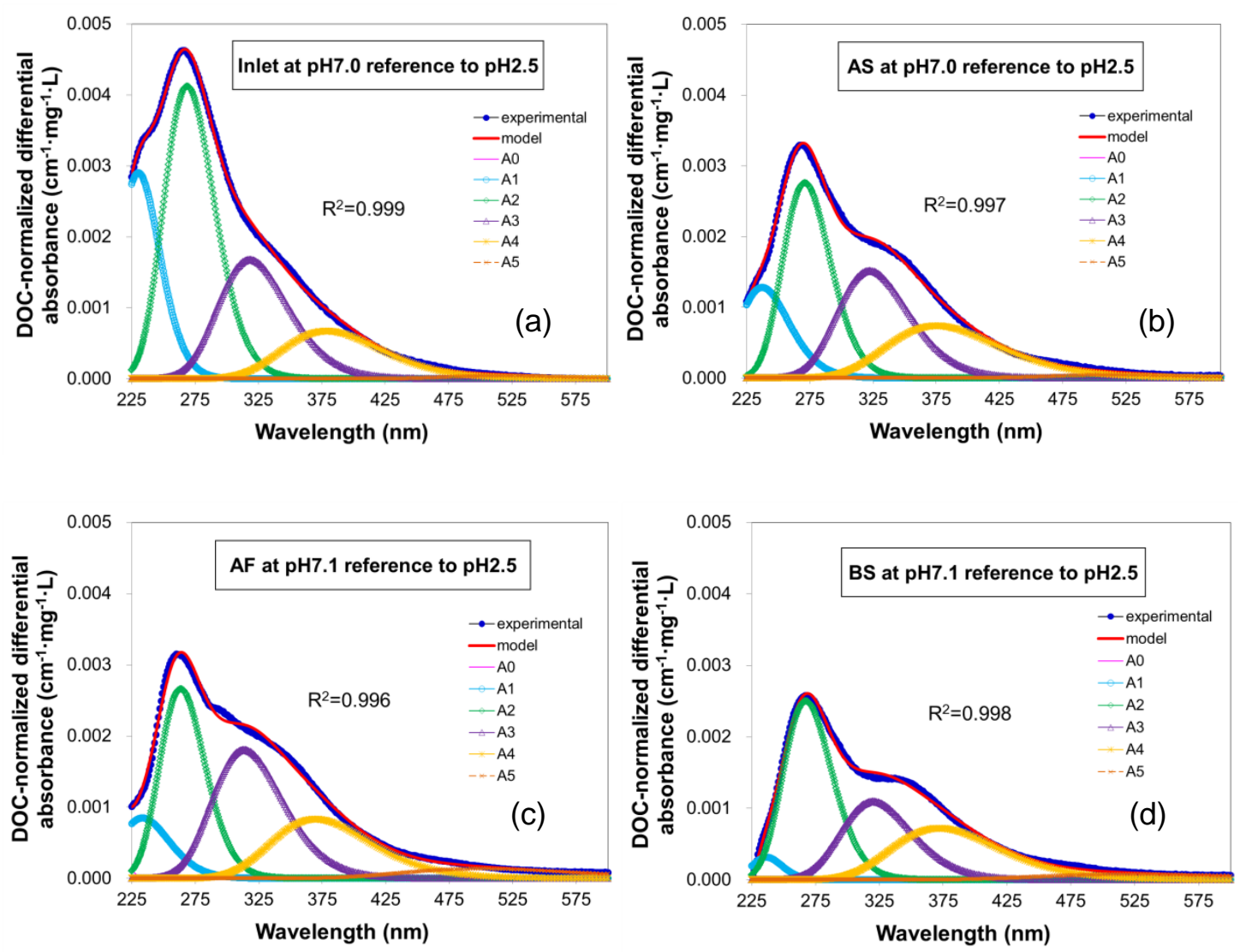

533

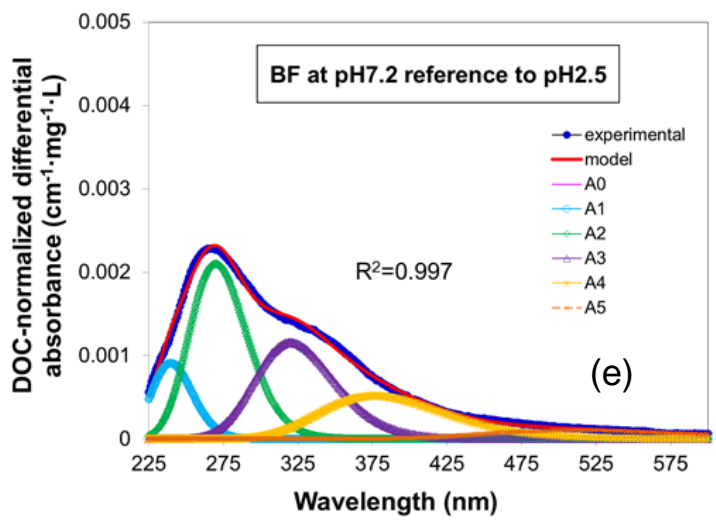

Figure 6. Gaussian band fitting of the differential DOM spectra in the influent and filtered $(\mathrm{BF}, \mathrm{e})$ water at $\mathrm{pH}$ about 7.0 referred to $\mathrm{pH} 2.5$. 

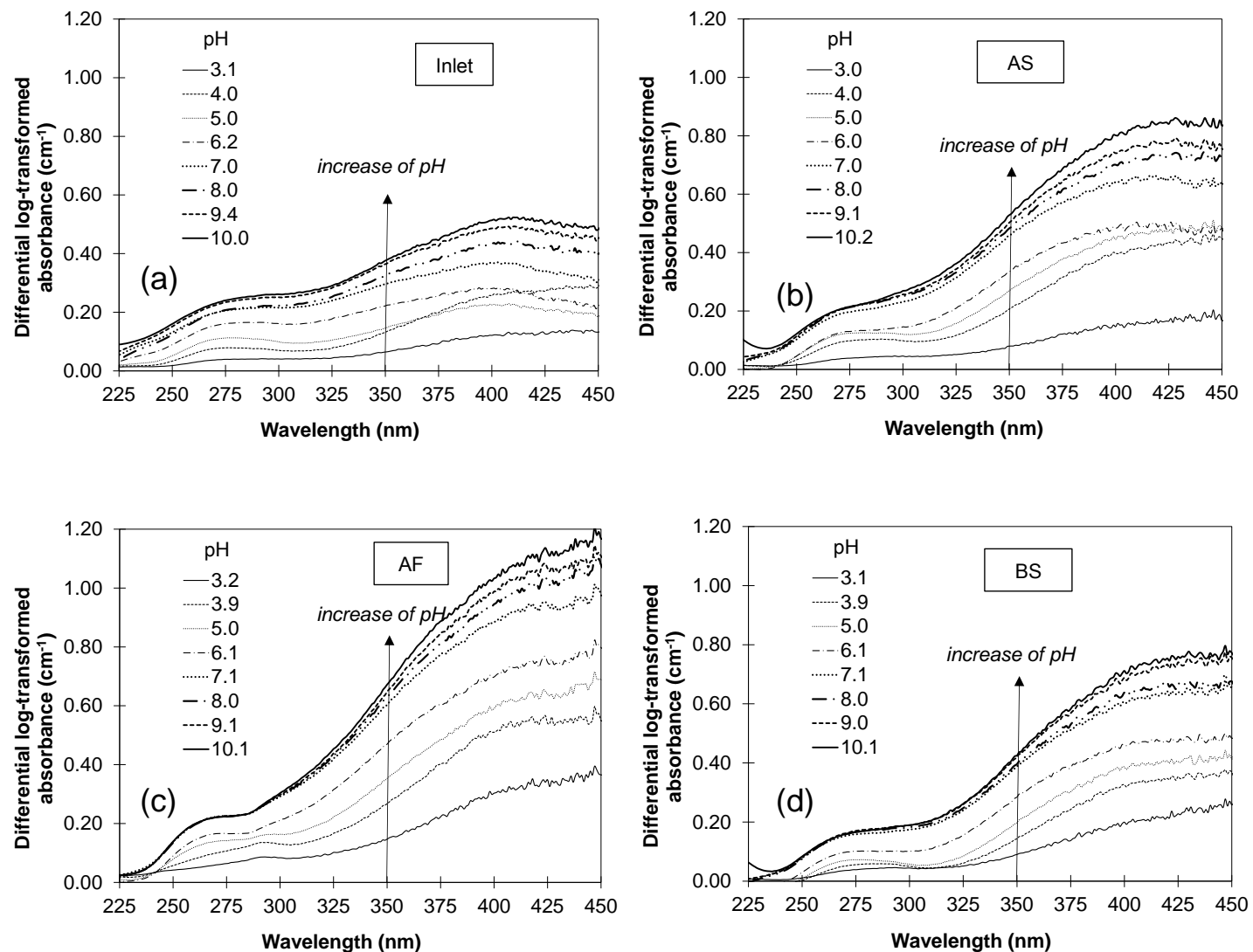

540

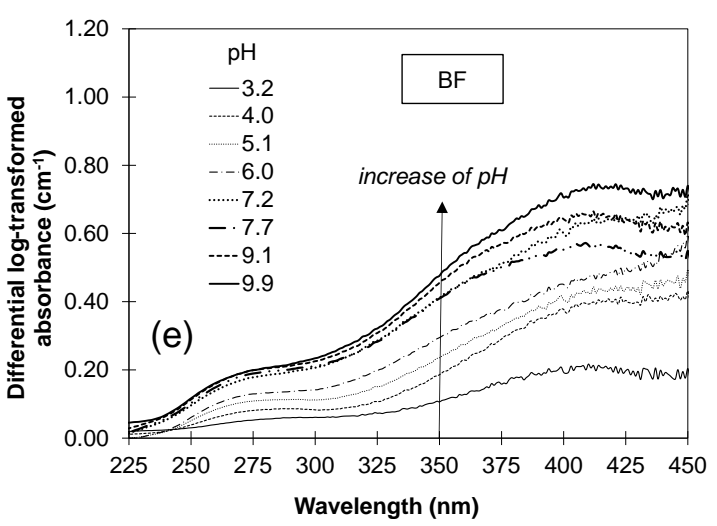

541

Figure 7. Log-transformed differential absorbance spectra of normalized differential absorbance of DOM in the influent (inlet, a), Plant A settled (AS, b)

544 and filtered (AF, c), and Plant B settled (BS, d) and filtered (BF, e) water. 


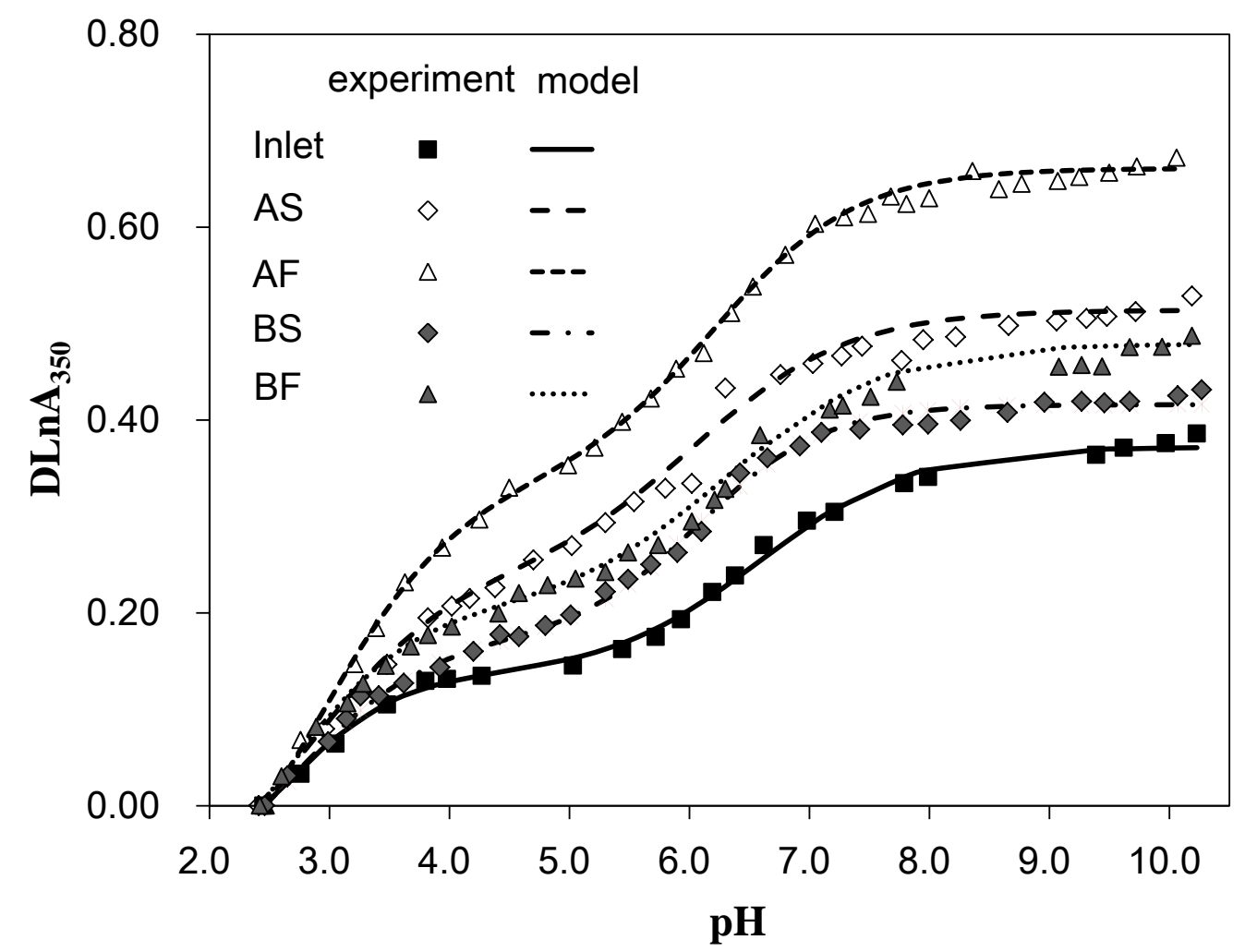

548 Figure 8. Comparison of effects of $\mathrm{pH}$ on the differential log-transformed 549 absorbance at wavelength $350 \mathrm{~nm}$ and their NICA-based fitting for DOMs in 550 RO isolates of influent (inlet), Plant A settled (AS) and filtered (AF ) and Plant 551 B settled (BS) and filtered (BF) water. 


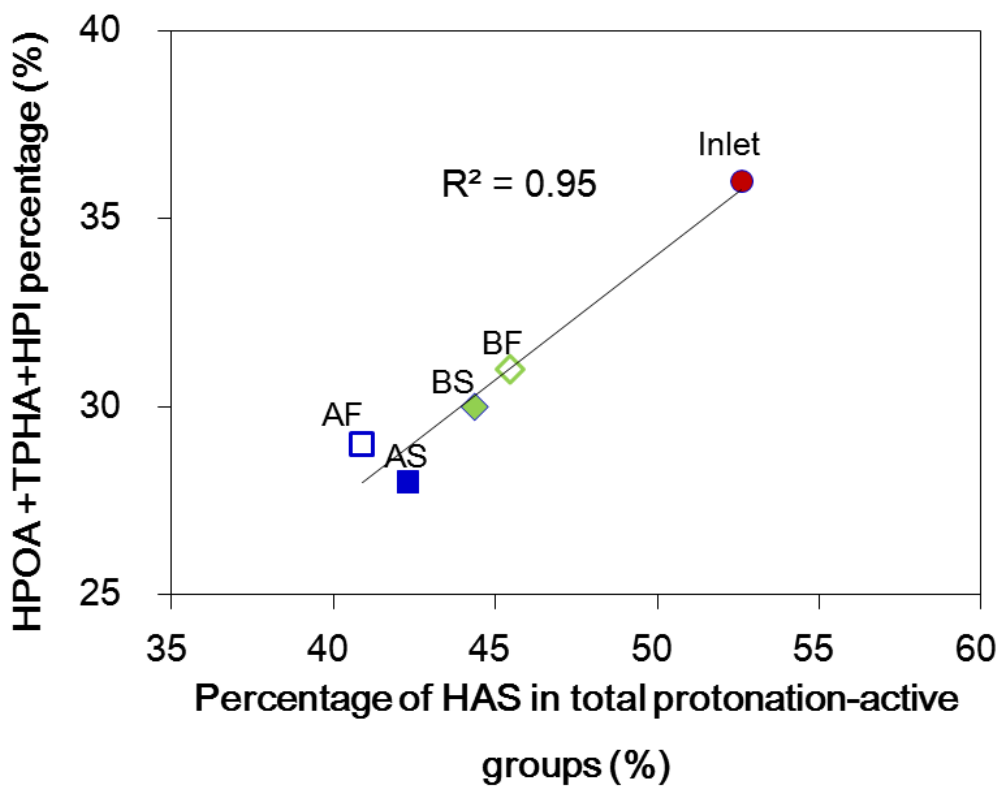

552 Figure 9. Correlation between the percentage of HAS in total 554 protonation-active groups vs. HPOA, THPA and HPI. DOMs in RO isolates of 555 influent (inlet), Plant A settled (AS) and filtered (AF), and Plant B settled (BS) 556 and filtered (BF) water. 


\section{Graphical Abstract}

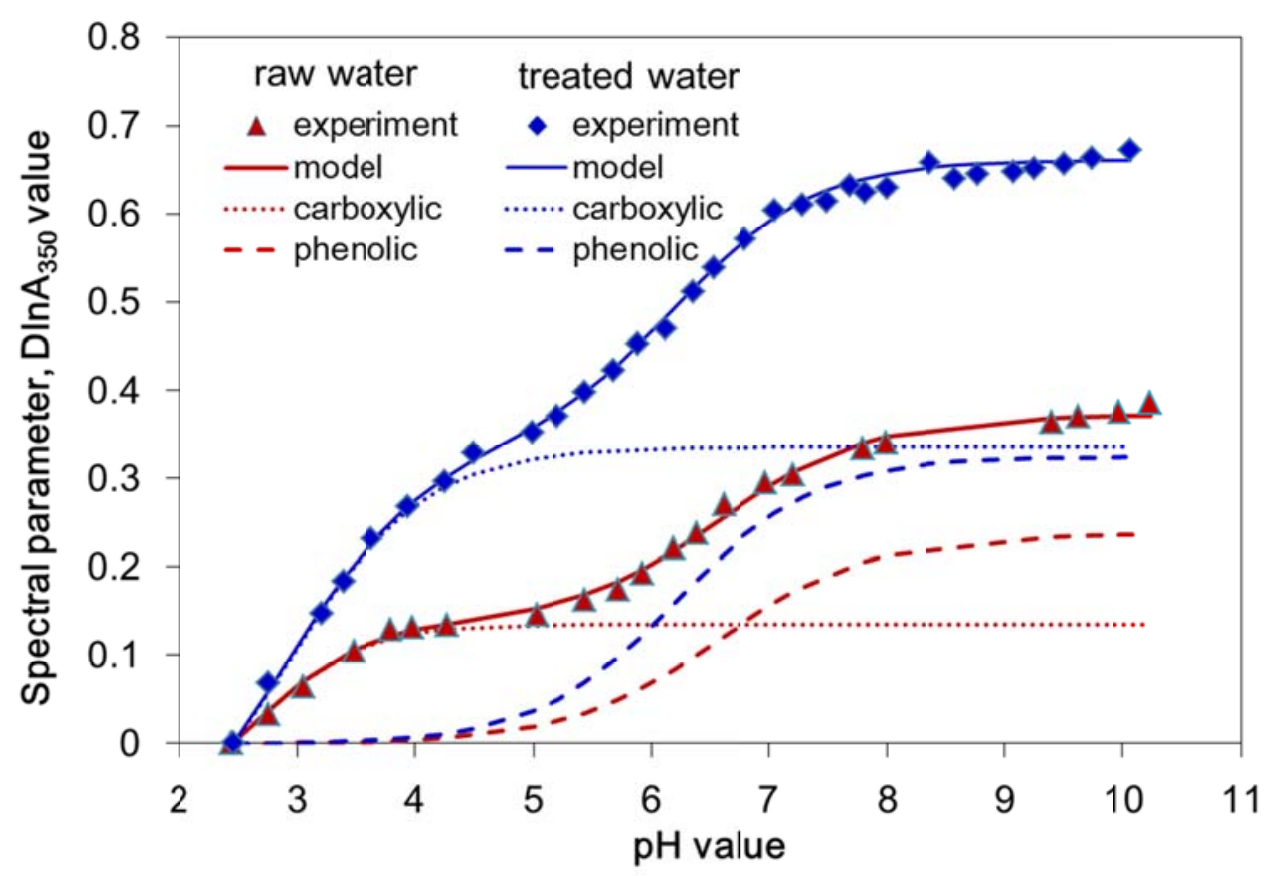

